Research Article

\title{
Study on Fluid-Structure Interaction of Flexible Membrane Structures in Wind-Induced Vibration
}

\author{
Fangjin Sun, ${ }^{1,2}$ Donghan Zhu, ${ }^{3}$ Tiantian Liu, ${ }^{3}$ and Daming Zhang $\mathbb{D}^{4,5}$ \\ ${ }^{1}$ Guangxi Key Laboratory of Geomechanics and Geotechnical Engineering, Guilin 541004, China \\ ${ }^{2}$ College of Civil Engineering and Architecture, Guilin University of Technology, Guilin 541004, China \\ ${ }^{3}$ College of Civil Engineering, Liaoning Technical University, Fuxin 123000, China \\ ${ }^{4}$ Guangxi Key Laboratory of Embedded Technology and Intelligence System, Guilin 541006, China \\ ${ }^{5}$ College of Information Science and Engineering, Guilin University of Technology, Guilin 541006, China \\ Correspondence should be addressed to Daming Zhang; e_horse@163.com
}

Received 13 November 2020; Revised 11 January 2021; Accepted 10 March 2021; Published 18 March 2021

Academic Editor: Alvaro Cunha

Copyright $\odot 2021$ Fangjin Sun et al. This is an open access article distributed under the Creative Commons Attribution License, which permits unrestricted use, distribution, and reproduction in any medium, provided the original work is properly cited.

\begin{abstract}
A strongly coupled monolithic method was previously proposed for the computation of wind-induced fluid-structure interaction of flexible membranous structures by the authors. How to obtain the accurate solution is a key issue for the strongly coupled monolithic method. Projection methods are among the commonly used methods for the coupled solution. In the work here, to impose initial pressure boundary conditions implicitly defined in the original momentum equations in classical projection methods when dealing with large-displacement of membranous structures, a modified factor is introduced in corrector step of classical projection methods and a new modified projection method is obtained. The solution procedures of the modified projection method aimed at strongly coupled monolithic equations are given, and the related equations are derived. The proposed method is applied to the computation of a two-dimensional fluid-structure interaction benchmark case and wind-induced fluid-structure interaction of a three-dimensional flexible membranous structure. The performance and efficiency of the modified projection method are evaluated. The results show that the modified projection methods are valid in the computation of wind-induced fluid-structure interaction of flexible membranous structures, with higher accuracy and efficiency compared with traditional methods. The modified value has little effects on the computation results whereas iteration times has significant effects. Computation accuracy can be improved greatly by increasing iteration times with less increase in computation time and little effects on stability with the modified projection method.
\end{abstract}

\section{Introduction}

The fluid-structure interaction of wind and membrane structure is one of the cutting-edge topics that have been widely concerned by researchers but have not been studied in depth. With the rapid improvement of computer hardware and software technology, numerical simulation methods have developed into an important tool for analyzing the fluid-solid coupling of wind and membrane structures. At present, numerical methods for studying fluid-structure interaction in wind vibration of membrane structures include weak coupling partitioning method, strong coupling partitioning method, and strong coupling integral method [1]. The weakly coupled partitioned method is to solve the fluid control equation and the structural control equation separately in each time step, and then the aerodynamic load acting on the fluid domain model is transferred to the structural domain model through the interface between the fluid and the structure, so as to predict the displacement of the structure. The displacement of the structure is then transferred to the fluid domain as a new load, and the process is repeated until the result converges to the specified value. The strongly coupled partitioned method is to add an iteration cycle based on solvers in the fluid domain and the structural domain, solving the nonlinear equations in each time step, and calculating the value of the variables in the whole field. These methods, respectively, integrate the governing equations explicitly and implicitly in time. In strongly coupled monolithic method, the governing equations for the flow and 
structure domains are discretized together, and the nonlinear system of equations is solved as a whole $[2,3]$.

Most scholars at home and abroad have studied the fluidstructure interaction in wind-induced vibration of membrane structures by strongly coupled partitioned method or weakly coupled partitioned method [3-8]. The research studies on the computation of fluid-structure interaction problem by strongly coupled monolithic methods are still very limited $[9,10]$, but the strongly coupled monolithic method shows its advantages in terms of stability and accuracy. Typically, the implicit coupling is needed for the large-scale structure deformation to ensure the numerical stability of the FSI solver [11]. In order to account structural dynamics in an FSI system, previous studies successfully integrated finite-element-based structural solver with existing flow solvers. For example, Bhardwaj and Mittal [12] proposed an FSI solver by coupling a sharp-interface IB method and an open-source finite-element solver (Tahoe), using an implicit partitioned approach. Employing this solver, they validated the FSI benchmark, proposed by Turek and Hron; cylinder attains self-sustained oscillation in a laminar channel flow. Similarly, Tian et al. [13] proposed a versatile FSI solver which could handle large-scale FID of a flexible structure. They carried out several validations with established benchmarks and demonstrated the three-dimensional capability of the solver. Bailoor et al. [14] coupled a compressible flow solver with an open-source finite- element solver (Tahoe) to simulate blast loading on thin plates. Very recently, Furquan and Mittal [15] numerically studied two side-by-side flexible splitter plates attached to square cylinders using a deformingspatial-domain/stabilized space-time flow solver coupled with a finite-element open-source structural dynamics solver. The authors [16] derived the strong coupled monolithic equations for fluid-structure interaction calculation of wind and membrane structures and analyzed the fluid-structure interaction of wind vibration of a membrane structure with typical shapes. The results showed that the strongly coupled monolithic method has advantages in both accuracy and stability in computation of fluid-structure interaction. However, since the strongly coupled monolithic method needs nonlinear equations to represent the fluid-structure interaction system, it is usually necessary to employ the Newton-Raphson method to solve the equations after linearization. For solving the equations, a large amount of computation time is spent on the repeated integration of the Jacobian matrix and the Newton modification of the solution to the corresponding linear system. This often leads to a large amount of computation time, and how to solve the strongly coupled monolithic equations is among one of the key issues to be solved.

In the work here, considering the characteristics of undergoing large deformations of flexible membrane structures, the traditional projection method is modified to solve the previously proposed strongly coupled monolithic equations by the authors. Based on the classical projection method, the iterative correction of the pressure correction factor is introduced into the correction step to satisfy the pressure constraint conditions implicitly defined in the original momentum method. The solution procedures of the modified projection method aimed at strongly coupled monolithic equations are given, and the related equations are derived. The proposed modified projection method is used to calculate the classical two-dimensional fluid-structure benchmark problem. The wind-induced fluid-structure interaction effects of a three-dimensional flexible membrane structure are obtained employing the proposed method.

\section{Governing Equations and Boundary Conditions}

2.1. Fluid Governing Equations. The incompressible viscous fluid is governed by the Navier-Stokes equations, consisting of momentum conservation and continuity equation. The governing equations for the structure are described by a total Lagrange (TL) formulation and a large deformation theory. A linear elastic model is introduced to deal with the data transfer at the interface, which is governed by semidiscretized finite element equations. These equations can be found in Sun and $\mathrm{Gu}$ [16]. The fluid governing equations are Navier-Stokes equation (abbreviated as N-S equation) for incompressible viscosity, namely, the continuum equation and the momentum equation:

$$
\begin{gathered}
\nabla \cdot v_{f}=0 \\
\rho_{f}\left(\frac{\partial v_{f}}{\partial t}+v_{f} \cdot \nabla v_{f}\right)=\nabla \cdot \sigma_{f}+f_{f}^{B},
\end{gathered}
$$

where $v_{f}$ is the fluid flow velocity, $\nabla$ is the spatial gradient, $\rho_{f}$ is the fluid density, $\sigma_{f}$ is the fluid's full stress tensor (pressure and viscous force), $\sigma_{f}=\mu\left[\nabla v_{f}+\left(\nabla v_{f}\right)^{T}\right]-p l, \mu$ is the fluid viscosity, $p$ is the fluid pressure, and $f_{f}^{B}$ is the fluid volume force.

2.2. Structural Governing Equations. The membrane structure can be regarded as an hyperelastic model and is described by the total Lagrangian equation:

$$
\nabla \cdot \sigma_{f}+f_{s}=0
$$

The above equations are based on the expression of the initial undeformed configuration, where $\sigma_{f}$ is the stress tensor of Piola-Lagrange, which is the stress with respect to the deformation configuration of the undeformed surface.

2.3. Fluid and Structure Coupling. A linear elastic model is introduced to deal with the deformation of the fluid domain, solve the data transfer problem at the interface of the fluid domain and the structural domain, and realize the coupling of fluid and structure. The equation of the linear elastic model is

$$
\begin{aligned}
\nabla \cdot \sigma_{l}^{\mathrm{LE}} & =0 \\
E^{\mathrm{LE}} & =\frac{1}{2}\left[h^{\mathrm{LE}}+\left(h^{\mathrm{LE}}\right)^{T}\right], \\
\sigma_{p(i j)}^{(\mathrm{LE})} & =\sigma_{c(i j)}^{\mathrm{LE}}+\vartheta_{(i j k l)}^{\mathrm{LE}} E_{k l}^{(\mathrm{LE})},
\end{aligned}
$$

where $\sigma_{l}^{\mathrm{LE}}$ is the linear elastic model Piola-Lagrange stress tensor; $E^{\mathrm{LE}}$ is the linear elastic model Green-Lagrange strain tensor; and $\sigma_{\rho}^{\mathrm{LE}}$ is the linear elastic model Piola-Kirchoff stress tensor. The boundary conditions of the above equation are as follows: 


$$
u_{\mathrm{LE}}^{\mathrm{FSI}}=0 \mathrm{on} \Gamma_{\mathrm{FSI}} \text {, }
$$

where $u_{\mathrm{LE}}^{\mathrm{FSI}}$ represents the displacement of the linear elastic model on the interface and $\Gamma_{\mathrm{FSI}}$ represents the fluid-solid interface; that is, the elastic displacement on the interface is zero. The coupling condition of fluid and structure at the interface is

$$
\begin{aligned}
u_{\mathrm{LE}}^{\mathrm{FSI}} & =u_{s}^{\mathrm{FSI}}, \text { on } \Gamma_{\mathrm{FSI}}, \\
v_{f}^{\mathrm{FSI}} & =v_{s}^{\mathrm{FSI}}, \text { on } \Gamma_{\mathrm{FSI}}, \\
\sigma_{c}^{\mathrm{FSI}} \cdot n_{s}+\sigma_{f}^{\mathrm{FSI}} \cdot n_{f} & =0 \mathrm{on} \Gamma_{\mathrm{FSI}},
\end{aligned}
$$

where $v_{s}$ is the structural velocity, $u_{s}$ is the unknown structural displacement, $\sigma_{c}$ is the Cauchy stress, $\sigma_{c}=F \sigma_{p} F^{T} / J, J=\operatorname{det}(F)$ is the Jacobian determinant, and $n_{s}=-n_{f}, n_{f}, n_{s}$ is the unit vector in the direction of the external normal of the fluid boundary.

\subsection{The Strongly Coupled Global Equation for a Fluid-Solid} Coupled System. The strongly coupled monolithic equations $f_{\text {FSI }}$ of the FSI in wind-induced fluid-structure interaction of flexible membranous structures read

$$
\begin{aligned}
f_{F} & =\int_{\Omega^{f}}\left(\omega \nabla \cdot v_{f}+-\zeta \cdot \rho_{f}\left(\frac{\partial v_{f}}{\partial t}+v_{f} \cdot \nabla v_{f}\right)-\nabla \zeta: \sigma_{f}+\zeta \cdot f_{f}\right) d Q=0, \\
f_{S} & =\int_{\Omega_{0}^{s}}\left(\nabla \eta: \sigma_{l}-\eta \cdot f\right)_{s} d \mathrm{Q}=0, \\
f_{\mathrm{LE}} & =\int_{\Omega_{0}^{s}}\left(\nabla \eta: \sigma_{l}^{\mathrm{LE}}\right) d \Omega=0, \\
f_{F}^{\mathrm{FSI}} & =\int_{\Gamma_{v}^{J} \cup \Gamma_{\mathrm{FSI}}} \zeta \cdot\left(\sigma_{f} \cdot n_{l}^{f}\right) d \Gamma=0, \\
f_{S}^{\mathrm{FSI}} & =\int_{\Gamma_{v 0}^{S} \cup \Gamma_{\mathrm{FSIO}}}\left(\left(\eta \cdot n_{0}^{s}\right): \sigma_{l}\right) d \Gamma+\int_{\Gamma_{v 0}^{s} \cup \Gamma_{\mathrm{FSI}}}\left(\left(\eta \cdot n_{0}^{S}\right): \sigma_{l}\right) d \Gamma=0,
\end{aligned}
$$

where the unknowns of the monolithic equations are the fluid velocity $v_{f}$, pressure $p$, linear elastic model displacement $u^{\mathrm{ES}}$, and structural displacement $u_{s}$.

Finally, along the interface, linear elastic model displacements are implicitly imposed to be equal to structural displacements in a strong sense:

$$
u^{\mathrm{ES}}=u_{s\left(\mathrm{on}^{\mathrm{I}} \mathrm{I}\right.} \text {. }
$$

The monolithic equations of the fluid-structure are solved using projection methods, which are commonly used methods for the coupled solution. But for large-displacement problems for flexible membranous structures, to impose initial pressure boundary conditions implicitly defined in the original momentum equations in classical projection method it is weak to obtain an accurate solution. Thus, the key issue here is how to modify the classical projection method considering large-displacement of membranous structures to obtain an accurate solution of the strongly coupled monolithic equations.

\section{Solution to Strongly Coupled Monolithic Equations}

The projection method in fluid dynamics is a common method for solving the nonlinear coupling equations. The projection method is generally divided into two steps of a prediction step and a correction step. For details of the corrector and prediction steps, please refer to reference [17]. Generally, the additional velocity calculated in the prediction step does not satisfy the nondispersion condition. For this reason, iterative correction is performed in the correction step so that the constraint conditions are satisfied. Although the traditional projection method can solve the pressure field and velocity field separately, it cannot satisfy the initial boundary conditions of pressure that are implicitly defined in the original momentum method, especially for the large deformation problem [18]. Therefore, this paper introduces the pressure correction factor into the projection method to solve the above problems.

First, the initial velocity pressure is set before the iterative calculation $\left(\mathbf{v}^{0}, p^{0}, \mathbf{u}^{0}\right)$, and $\widetilde{v}^{0}=\mathbf{v}^{0}, p_{a, \text { proj }}^{0}=0$, and the additional velocity field $\mathbf{v}_{a}^{k, n+1}$ at the moment of $(n+1)$ is solved by the following equation:

$$
\begin{aligned}
\frac{F_{F}}{\Delta t}\left(\widetilde{v}_{a}^{f, n+1}, \phi_{a}\right)_{\Omega}+f_{\mathrm{LE}}\left(\widetilde{v}_{a}^{f, n+1}, \phi_{a}\right)_{\Omega^{f}}+u_{s}\left(\widetilde{v}_{a}^{s, n+1}, \phi_{a}\right)_{\Omega^{s}} \\
\quad-\lambda_{1}\left(\left(\nabla \cdot \widetilde{v}_{a}^{f, n+1}\right)_{\pi^{\prime}}, \nabla \cdot \phi_{a}\right)_{\Omega} \\
=\frac{F_{S}}{\Delta t}\left(\widetilde{v}_{a}^{s, n+1}, \phi_{a}\right)_{\Omega}+\left(\eta p_{a, \mathrm{proj}}^{k, n+1}+\delta p_{a, p}^{n}, \nabla \cdot \phi_{a}\right)_{\Omega} \quad \forall \phi_{a} \in S_{a}(\Omega),
\end{aligned}
$$

where $p_{a, \text { proj }}^{k, n+1}$ is the projection term in the correction step, $\delta p_{a, p}^{n}$ is the pressure correction factor, which is used to apply 
the initial boundary conditions of the pressure to satisfy the original momentum equation, $\widetilde{v}_{a}$ is the additional speed in the prediction step, and $\lambda_{1}$ is the correction coefficient.

$$
\begin{aligned}
\mathrm{F}_{L E}\left(\widetilde{v}_{a}^{f}, \phi_{a}\right) & =u\left(\widetilde{v}_{a}^{f, n+1}, \phi_{a}\right)-\left(\rho^{f}\left(\nabla \cdot \phi_{a}\right) \widetilde{v}_{a}^{f, n+1}, \phi_{a}\right)-\mathbf{f}_{F}^{\mathrm{FSI}}, \\
u^{s}\left(\widetilde{v}_{a}^{s}, \psi_{a}\right) & =\Delta t u^{s}\left(\widetilde{v}_{a}^{s, n+1}, \phi_{a}\right)-\mathbf{f}_{S}^{\mathrm{FSI}} .
\end{aligned}
$$
time:

Update the pressure correction factor $\delta p_{a, p}^{n}$ at $(n+1)$

$$
\delta p_{a, p}^{k, n+1}=\delta p_{a, p}^{k-1, n+1}+\lambda_{2}\left(\nabla \cdot \widetilde{v}_{a}^{k-1, n+1}\right) .
$$

When the additional velocity field $\left\|\widetilde{v}_{a}^{k+1, n+1}-\widetilde{v}_{a}^{k, n+1}\right\|$ between two adjacent iteration steps is less than the specified tolerance, the calculation is stopped and the following steps are entered.

According to the calculated additional velocity field $\mathbf{v}_{a}^{k, n+1}$ at the moment of $(n+1)$, the following formula is used to calculate the projection term $p_{a, p r o j}^{n+1}$ in the projection step:

$$
\begin{gathered}
\partial t\left(\widetilde{V}_{a}^{f}, \varphi_{a}\right)+\partial t\left(\widetilde{V}_{a}^{s}, \varphi_{a}\right)+F_{\mathrm{LE}}\left(\widetilde{V}_{a}^{f}, \varphi_{a}\right)+u^{s}\left(\widetilde{V}_{a}^{s}, \varphi_{a}\right) \\
-\left(p_{a}^{f, k}, \nabla \cdot \varphi_{a}\right)-\left(\delta p_{a, k}^{f, k}, \nabla \cdot \varphi_{a}\right)=0 \quad \forall \phi_{a} \in S_{a}(\Omega), \\
\left(\nabla \delta p_{a, \text { proj }}^{n+1}, \nabla \varphi_{a}\right)=-\frac{\rho}{\Delta t}\left(\nabla \cdot \widetilde{v}^{n+1}, \varphi_{a}\right), \quad \forall \varphi_{a} \in \Omega .
\end{gathered}
$$

Finally, project the velocity predicted above into the nonscattered vector field space to obtain the velocity, pressure, and displacement of the nonlinear equations:

$$
\begin{aligned}
\mathbf{v}_{f} & =\widetilde{v}_{a}^{f, n+1}-\frac{\Delta t}{\rho^{f}} \nabla \delta \widetilde{p}_{a, \text { proj }}^{f, n+1} \text { in } \Omega^{f}, \\
p & =p_{h}^{n}+\delta \widetilde{p}_{h, p r o j}^{n+1}+\delta \widetilde{p}_{h, p r o j}^{n+1} \text { in } \Omega_{f}, \\
u_{s} & =u_{a}^{n}+\Delta t \mathbf{v}_{f}^{n+1} .
\end{aligned}
$$

\section{Examples}

4.1. Two-Dimensional Fluid-Structure Interaction. Here, a benchmark case of two-dimensional fluid-structure interaction [18] is studied by the proposed method, whose details are not described here. The settings and parameters are as follows: the density of fluid water in the experiment $\rho_{f}=1000 \mathrm{~kg} / \mathrm{m}^{3}$, the solid is similar to rubber material, and the geometric dimensions are shown in Figure 1. The test is divided into three tests: FSI1, FSI2, and FSI3. The FSI1 test is a steady state test, and the FSI2 and FSI3 tests are two-dimensional calculations of unsteady fluid-solid coupling. Here, the FSI2 and FSI3 tests are calculated, respectively. In the case of low Reynolds number, the calculated domain length $L=2.5 \mathrm{~m}$ and height $H=0.02 \mathrm{~m}$ in the test. The structural model consists of a rigid cylinder and an elastic rod at its rear right, the position of the center of the cylinder (from the position of the lower left corner of the calculation field) $\mathrm{C}$ is $(0.2,0.2)$, and the radius is 0.05 . The length of elastic structure bar is $L=3.5$ and the height is $H=0.02$. The lower right corner is $(0.6,0.19)$, and its left side is completely connected to the fixed cylinder. The position of the control point $A(t)$ of the whole structure is $A(0)=(0.6,0.2)$. Due to space limitations, only the calculation results of the simulated FSI3 test are given here.

$\lambda_{1} \approx 10^{4}$ is set, and the results obtained after multiple iterations are compared with the results using the Newton-Raphson solution method [16] and the original test results [19]. Figures 2 and 3, respectively, show the displacement histories in different directions of the elastic beam end node $A$ calculated in this paper, as well as the lift force and resistance acting on the cylinder and beam. It can be seen from the figure that the calculation results of the projection method with the modified factor introduced in this paper are very close to those of the Newton-Raphson method and the original test results, indicating the correctness of this method. In order to illustrate the solution efficiency of the method in this paper, Table 1 shows the $x$ direction displacement $\mathbf{d}_{x}$ and $y$-direction displacement $\mathbf{d}_{y}$ of the elastic beam end node $A$ calculated by this method with the error norm of different correction iterations, and it is compared with the results of other methods.

It can be seen from Table 1 that, compared with the traditional Newton-Raphson method, the error norm of the projection method with correction factor introduced in this paper is much smaller after the same number of iterations. Moreover, the method in this paper only needs to pass a small number of iterations to achieve a small error, which proves that the method of this paper has a fast convergence speed and a small error.

\subsection{Analysis of Wind-Induced Vibration Fluid-Structure In-} teraction of Three-Dimensional Flexible Membrane Structure. Taking the typical saddle-shaped membrane structure as an example, the above strong coupling overall method program is used to analyze and calculate the wind-induced vibration response. The calculation diagram of the membrane structure is shown in Figure 4, and its basic parameters are as follows: span $L=20 \mathrm{~m}$, height $H=5 \mathrm{~m}$, rise-span ratio $\mathrm{fl}$ $L=1 / 8$, pretension $T=2.0 \mathrm{kN} / \mathrm{m}$, film thickness $1 \mathrm{~mm}$, mass per unit area $g=1.25 \mathrm{~kg} / \mathrm{m}^{2}$, tensile stiffness is $E t=8.0 \times 105 \mathrm{~N} / \mathrm{m}$, shear stiffness $G t=1.2 \times 104 \mathrm{~N} / \mathrm{m}$, and Poisson's ratio $=0.3$. Because the performance of modified projection method is mainly studied in this paper, the influence of turbulence is not considered. Taking a typical three-dimensional saddle-shaped membrane structure as an example, the above strong coupling overall method and preprocessor are used to analyze and calculate the windinduced vibration response. The specific parameter settings in the calculation are the same as those in [16] and will not be repeated here.

Before the fluid-structure interaction computation, a dynamic analysis of the saddle membrane structure analyzed in the paper is carried out. We have got some grid pictures showing the first four vibration modes and frequencies results of the membrane structures, as shown in Figure 5. 


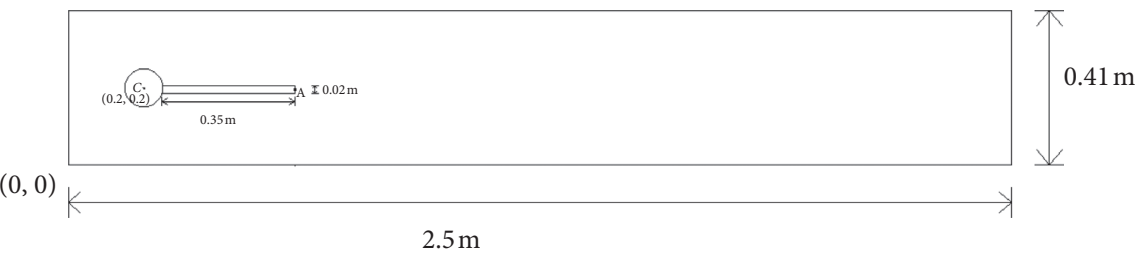

FIgURE 1: Geometrical dimensions of classical fluid-structure coupling problem.

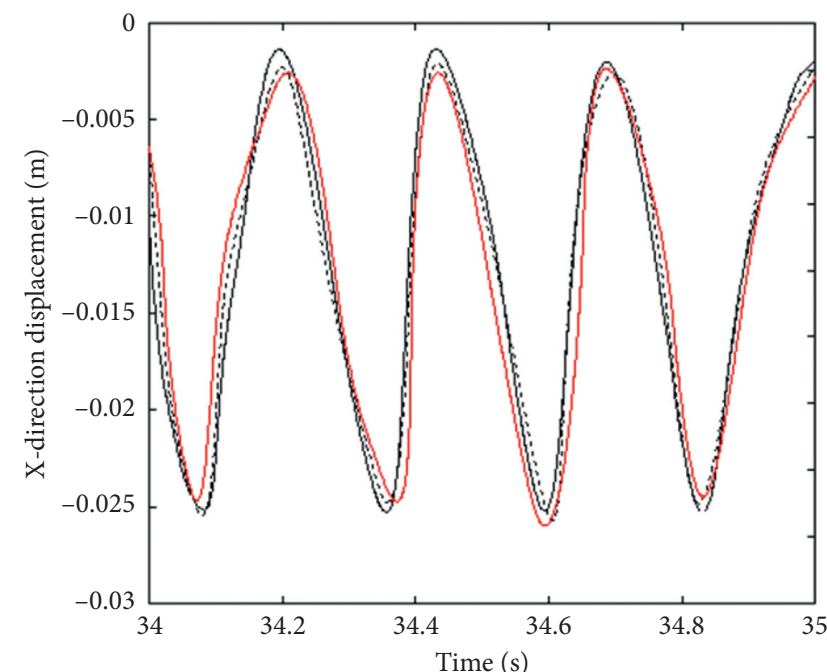

- Newton-Raphson method

..... Turek et al. (2006)

— Present method

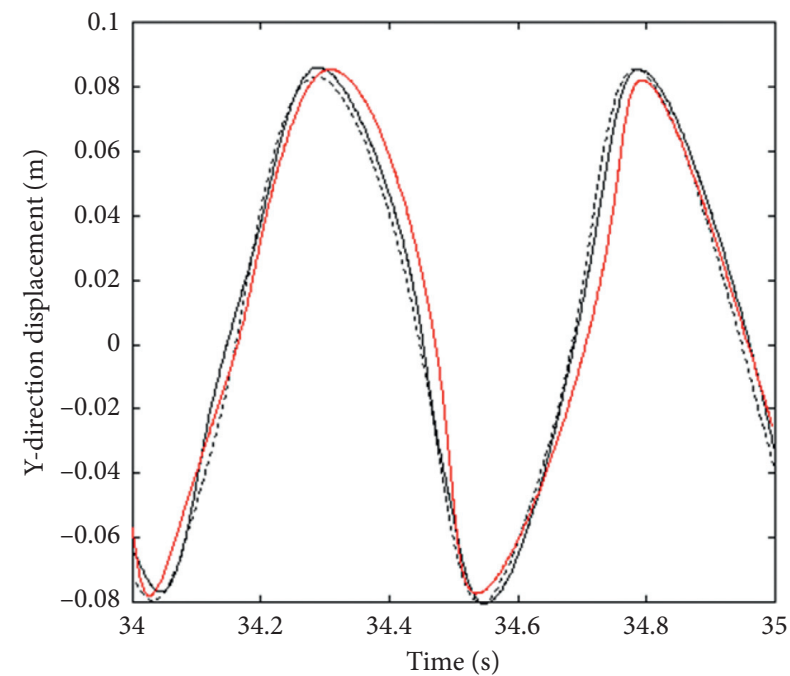

- Newton-Raphson method

..... Turek et al. (2006)

— Present method

(a)

(b)

Figure 2: Comparison of displacement history of elastic beam end node $A$ in different directions.

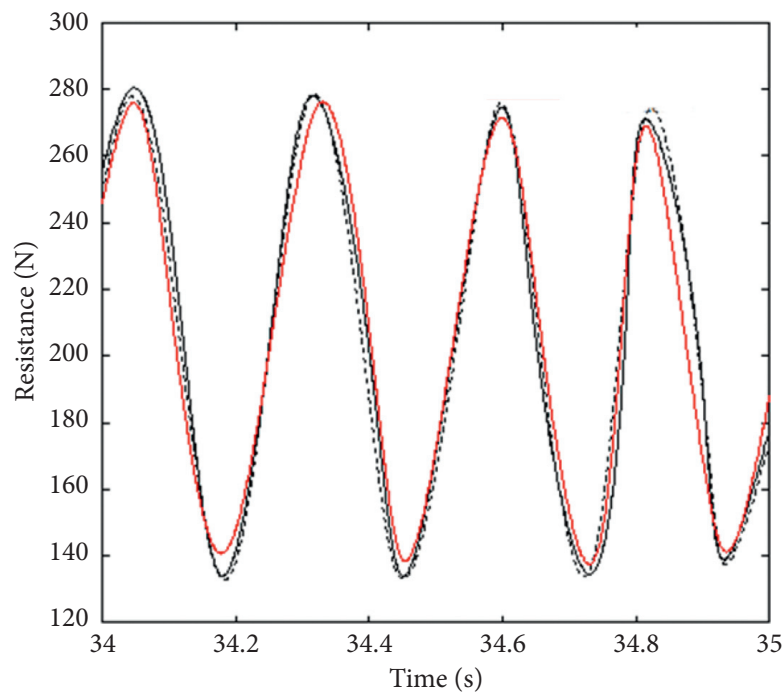

- Newton-Raphson method

..... Turek et al. (2006)

— Present method

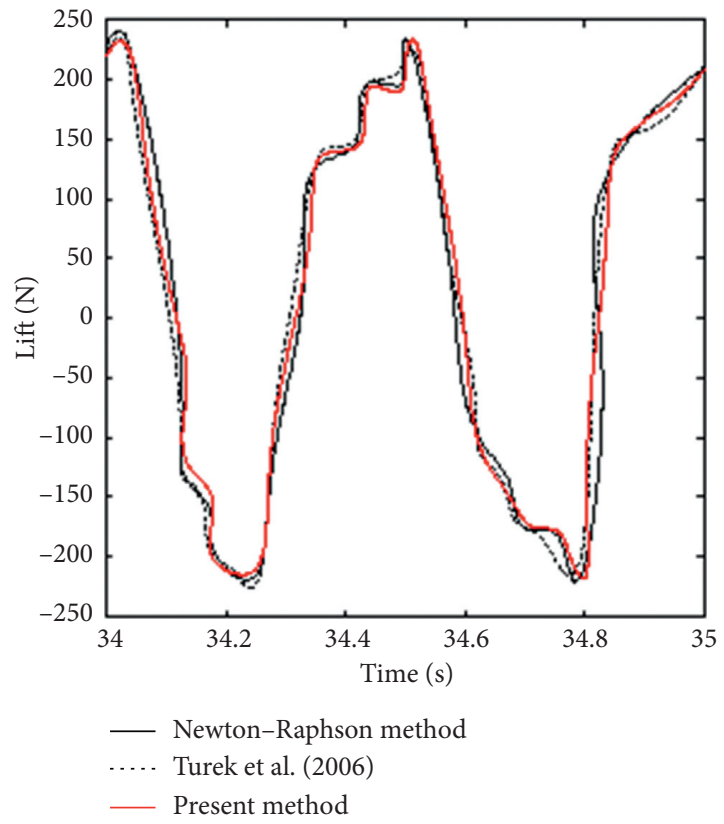

(b)

Figure 3: Comparison of lift and drag forces acting on cylinders and beams. 
Table 1: Comparison of error norm of elastic beam end node $A$.

\begin{tabular}{lccc}
\hline Number of iterations & Adapting methods & $\left\|\mathbf{d}_{x}-\mathbf{d}_{x}^{0}\right\|$ & $\left\|\mathbf{d}_{y}-\mathbf{d}_{y}^{0}\right\|$ \\
\hline \multirow{2}{*}{10} & Present method & $1.34 e-4$ & $2.46 e-4$ \\
& Newton-Raphson method & $2.65 e-2$ & $4.88 e-2$ \\
\hline \multirow{2}{*}{16} & Present method & $5.37 e-6$ & $6.44 e-4$ \\
\hline \multirow{2}{*}{22} & Newton-Raphson method & $3.37 e-4$ & $2.55 e-7$ \\
& Present method & $3.23 e-7$ & $4.34 e-4$ \\
\hline \multirow{2}{*}{28} & Newton-Raphson method & $1.22 e-4$ & $3.92 e-8$ \\
& Present method & $4.17 e-8$ & $2.79 e-5$ \\
\hline \multirow{2}{*}{34} & Newton-Raphson method & $3.23 e-5$ & $1.94 e-8$ \\
& Present method & $2.61 e-8$ & $1.04 e-5$ \\
\hline
\end{tabular}

Note: $\mathbf{d}_{x}^{0}$ and $\mathbf{d}_{y}^{0}$ are analytical displacements.

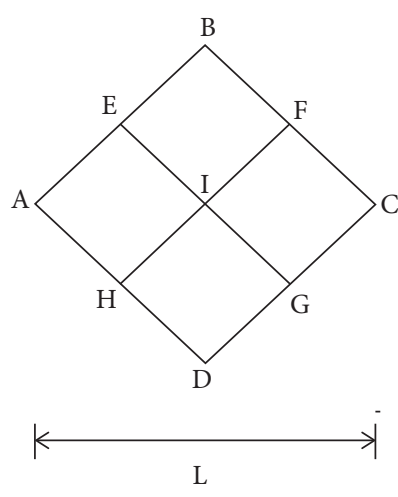

(a)
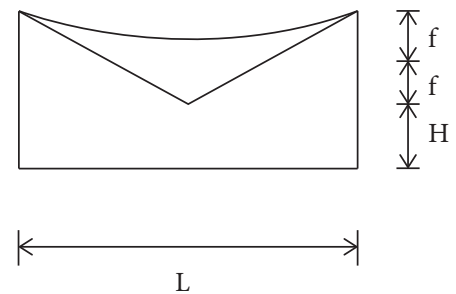

$\mathrm{L}$

Figure 4: Geometry of saddle membrane structure.

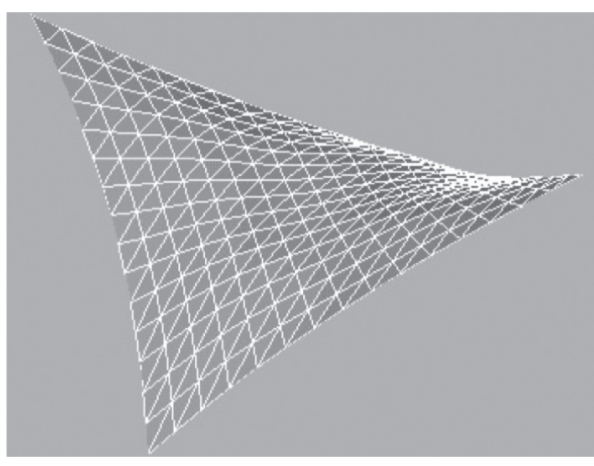

(a)

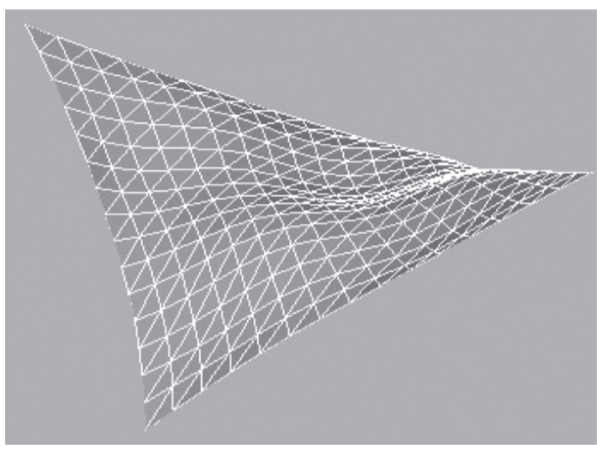

(c)

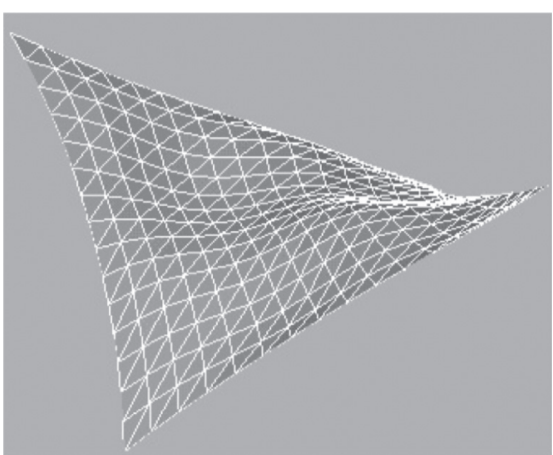

(b)

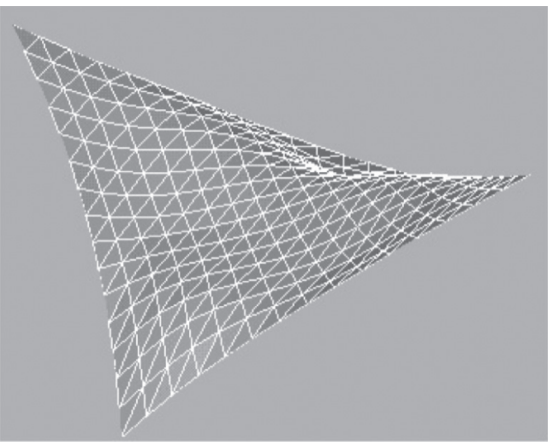

(d)

Figure 5: First four vibration modes of the saddle membrane. (a) First vibration mode $(f=2.87 \mathrm{~Hz})$. (b) Second vibration mode $(f=3.96 \mathrm{~Hz})$. (c) Third vibration mode $(f=4.05 \mathrm{~Hz})$. (d) Fourth vibration mode $(f=4.31 \mathrm{~Hz})$. 


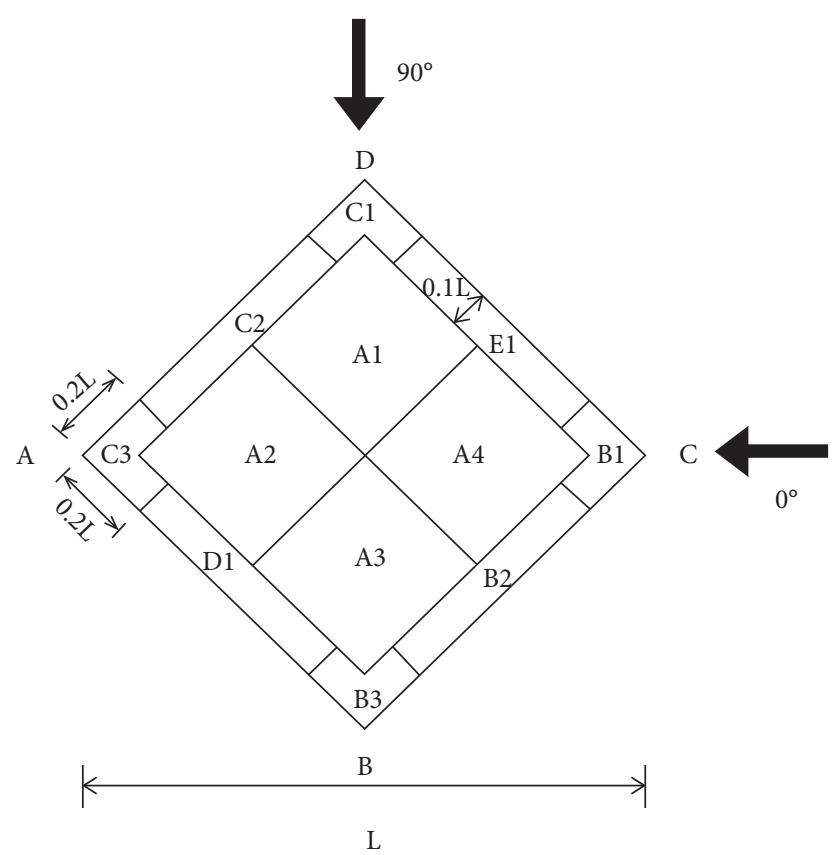

Figure 6: Partitioned diagram of saddle membrane structure.

In order to illustrate the computational efficiency and performance of the modified projection method in this paper, firstly, the influence of the modified value in the modified projection method on the wind pressure coefficient of the membrane structure is analyzed, and the partition of the saddle membrane structure is shown in Figure 6.

Table 2 shows the error norm when comparing the zonal wind pressure coefficient $\left(0^{\circ}\right.$ wind angle) of the Newton-Raphson method with different correction values selected in this paper. The zonal wind pressure coefficient of the Newton-Raphson method comes from literature [16], and the number of iterations is 10 .

After analyzing Table 2, the following conclusions can be drawn:

(1) The zonal wind pressure coefficient obtained by the modified projection method in this paper is very close to that obtained by the Newton-Raphson method, which proves the correctness of the calculation method for the three-dimensional film structure wind-induced fluid-structure coupling.

(2) The value of the corrected value $\lambda_{1}$ of the modified projection method in this paper does not have a great influence on the wind pressure coefficient. It can be seen that the error norm of the wind pressure coefficient does not change significantly with the increase of the corrected value $\lambda_{1}$. However, it is found that the increase of the correction value will lead to a significant increase in computer time and increase the risk of computational instability. Therefore, it is recommended that the correction value should be within a certain suitable range.
Table 3 shows the influence of the change of iteration times on the wind pressure coefficient, and the error norm change of the zonal wind pressure coefficient compared with the Newton-Raphson method at $0^{\circ}$ wind direction angle is also calculated. Modifier value $\lambda_{1} \approx 10^{3}$.

It can be seen from Table 3 that the calculation accuracy of the modified projection method is relatively high, and the number of iterations has an important influence on the wind pressure coefficient: the larger the number of iterations, the smaller the error norm of the wind pressure coefficient, and the more accurate the result. But it is important to pay attention to the balance between the accuracy of the calculation results and the calculation time. In the calculation, it was found that the average number of iterations increased by about $20 \%$, the calculation accuracy increased by about $19 \%$, and the calculation time increased by about $7 \%$. It shows that the modified projection method is used to calculate the windinduced fluid-solid coupling wind pressure coefficient of the membrane structure. The speed of calculation accuracy is higher than the number of iterations and time-consuming calculation. Therefore, if a more accurate result is needed, it can be achieved by increasing the number of iterations if the computational hardware conditions permit.

At the same time, in order to further illustrate the calculation accuracy and efficiency of the modified projection method in this paper, this paper also compares the windinduced vibration response of the first 40 s of the point in the membrane structure calculated by different solution methods under different grid numbers. With regard to the relative residual error $R$ and $T$ (hour) when using the same number of iterations, where the correction value of the modified projection method $\lambda_{1} \approx 10^{3}$, the comparison results are shown in Table 4 and Figure 7. 
TABLE 2: The effect of different correction values on the average wind pressure coefficient (mean).

\begin{tabular}{|c|c|c|c|c|}
\hline \multirow[t]{2}{*}{ Partition } & \multirow[t]{2}{*}{ Newton-Raphson method } & \multicolumn{3}{|c|}{$\begin{array}{l}\text { The method in this paper takes the error norm of different } \\
\text { correction values }\end{array}$} \\
\hline & & $10 e 3$ & $10 e 4$ & $10 e 5$ \\
\hline A1 & -0.52 & $2.14 e-4$ & $1.22 e-4$ & $3.23 e-4$ \\
\hline A2 & -0.34 & $1.49 e-4$ & $2.03 e-5$ & $2.98 e-4$ \\
\hline A3 & -0.22 & $3.23 e-4$ & $1.29 e-4$ & $1.22 e-5$ \\
\hline A4 & -0.54 & $2.33 e-5$ & $1.78 e-4$ & $3.22 e-4$ \\
\hline B1 & -1.56 & $3.21 e-4$ & $1.44 e-5$ & $2.03 e-4$ \\
\hline B2 & -1.35 & $0.92 e-4$ & $1.68 e-4$ & $2.43 e-4$ \\
\hline B3 & -0.86 & $2.31 e-4$ & $1.09 e-5$ & $1.65 e-4$ \\
\hline $\mathrm{C} 1$ & -0.76 & $2.33 e-4$ & $1.02 e-5$ & $2.11 e-5$ \\
\hline $\mathrm{C} 2$ & -0.15 & $0.94 e-4$ & $3.76 e-4$ & $2.76 e-5$ \\
\hline $\mathrm{C} 3$ & -0.13 & $3.66 e-4$ & $1.34 e-5$ & $3.22 e-4$ \\
\hline D1 & 0.18 & $1.75 e-5$ & $0.54 e-5$ & $3.18 e-4$ \\
\hline E1 & -1.28 & $0.43 e-5$ & $1.29 e-4$ & $0.87 e-5$ \\
\hline
\end{tabular}

TABLE 3: The effect of different iteration times on the average wind pressure coefficient (mean).

\begin{tabular}{|c|c|c|c|c|c|}
\hline \multirow{2}{*}{ Partition } & \multirow{2}{*}{ Newton-Raphson method } & \multicolumn{4}{|c|}{ The error norms of different iterations in this method } \\
\hline & & 10 & 18 & 26 & 34 \\
\hline A1 & -0.52 & $2.14 e-4$ & $1.22 e-5$ & $0.46 e-7$ & $0.98 e-8$ \\
\hline $\mathrm{A} 2$ & -0.34 & $1.49 e-4$ & $1.25 e-6$ & $3.23 e-7$ & $4.12 e-8$ \\
\hline A3 & -0.22 & $3.23 e-4$ & $2.75 e-5$ & $0.87 e-7$ & $3.23 e-8$ \\
\hline A4 & -0.54 & $2.33 e-5$ & $2.37 e-6$ & $1.75 e-7$ & $2.57 e-8$ \\
\hline B1 & -1.56 & $3.21 e-4$ & $4.45 e-5$ & $3.54 e-7$ & $0.85 e-8$ \\
\hline $\mathrm{B} 2$ & -1.35 & $0.92 e-4$ & $3.76 e-5$ & $4.34 e-7$ & $2.53 e-8$ \\
\hline B3 & -0.86 & $2.31 e-4$ & $3.93 e-5$ & $1.86 e-8$ & $5.94 e-9$ \\
\hline $\mathrm{C} 1$ & -0.76 & $2.33 e-4$ & $5.23 e-6$ & $3.75 e-7$ & $5.74 e-8$ \\
\hline $\mathrm{C} 2$ & -0.15 & $0.94 e-4$ & $3.76 e-6$ & $2.76 e-7$ & $0.45 e-8$ \\
\hline C3 & -0.13 & $3.66 e-4$ & $1.34 e-5$ & $3.22 e-6$ & $1.54 e-7$ \\
\hline D1 & 0.18 & $1.75 e-5$ & $3.24 e-6$ & $4.65 e-7$ & $2.95 e-8$ \\
\hline E1 & -1.28 & $0.43 e-5$ & $4.73 e-6$ & $1.76 e-7$ & $4.62 e-8$ \\
\hline
\end{tabular}

TABLE 4: Relative residuals and time-consuming under different grid numbers.

\begin{tabular}{|c|c|c|c|c|}
\hline \multirow{2}{*}{ Total number of grids (ten thousand) } & \multicolumn{2}{|c|}{ Newton-Raphson method } & \multicolumn{2}{|c|}{ Present method } \\
\hline & $R$ & $T$ & $R$ & $T$ \\
\hline 31 & $4.419 \times 10^{-5}$ & 149 & $1.122 \times 10^{-5}$ & 84 \\
\hline 33 & $4.204 \times 10^{-5}$ & 178 & $8.329 \times 10^{-6}$ & 93 \\
\hline 36 & $3.986 \times 10^{-5}$ & 209 & $5.788 \times 10^{-6}$ & 103 \\
\hline 39 & $3.795 \times 10^{-5}$ & 247 & $3.866 \times 10^{-6}$ & 114 \\
\hline
\end{tabular}

The following can be seen from Table 4 and Figure 7:

(1) Under the same grid accuracy and iteration times, regardless of whether the traditional Newton-Raphson method or the method in this paper is used, as the grid refinement increases, the relative residuals of the calculation are gradually reduced, and the computation time is also increased. However, the relative residual error and machine time consumption of the modified projection method in this paper are lower than those of the traditional Newton-Raphson method, which indicates a higher accuracy and efficiency of the proposed method.
(2) For Newton-Raphson method, when the grid accuracy increases by about $10 \%$ on average, the accuracy increases by about $5 \%$, while the computation time increases by about $20 \%$ on average, and the stability is basically not affected, indicating that the fineness of grid division has little effect on the accuracy and stability.

(3) When using the modified projection method in this paper, when the grid accuracy is increased by about $10 \%$ on average, the calculation accuracy is increased by about $30 \%$ on average, while the time spent on the machine is only increased by about $10 \%$ on average, and the stability is basically not affected. It shows 


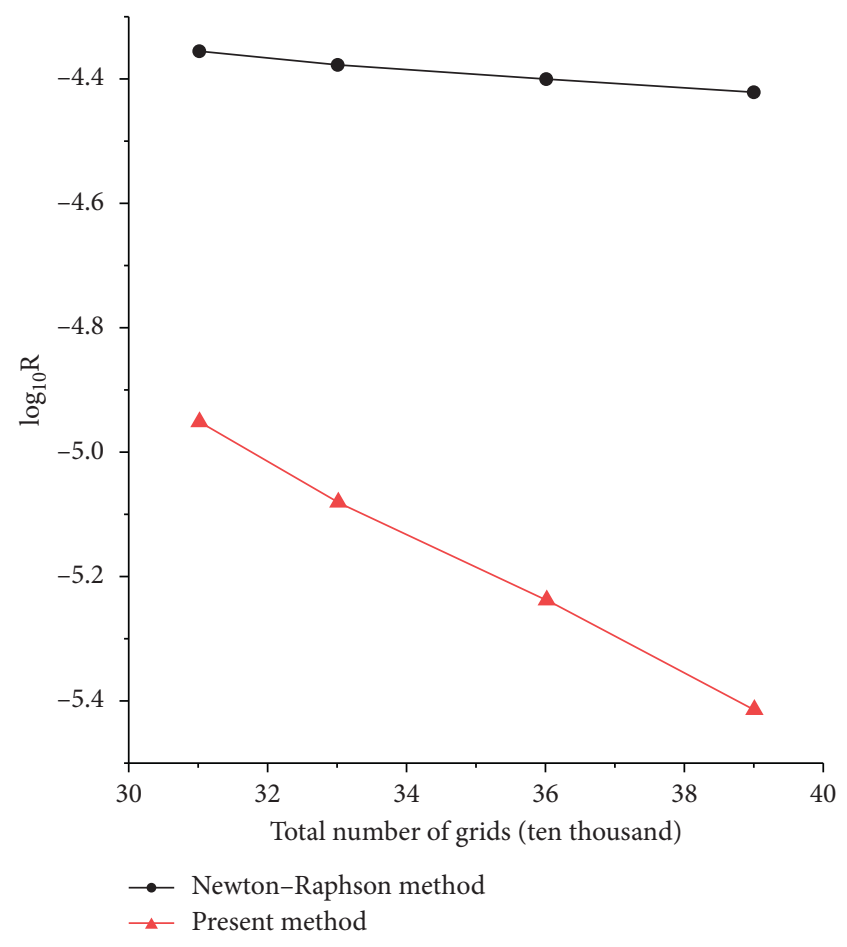

(a)

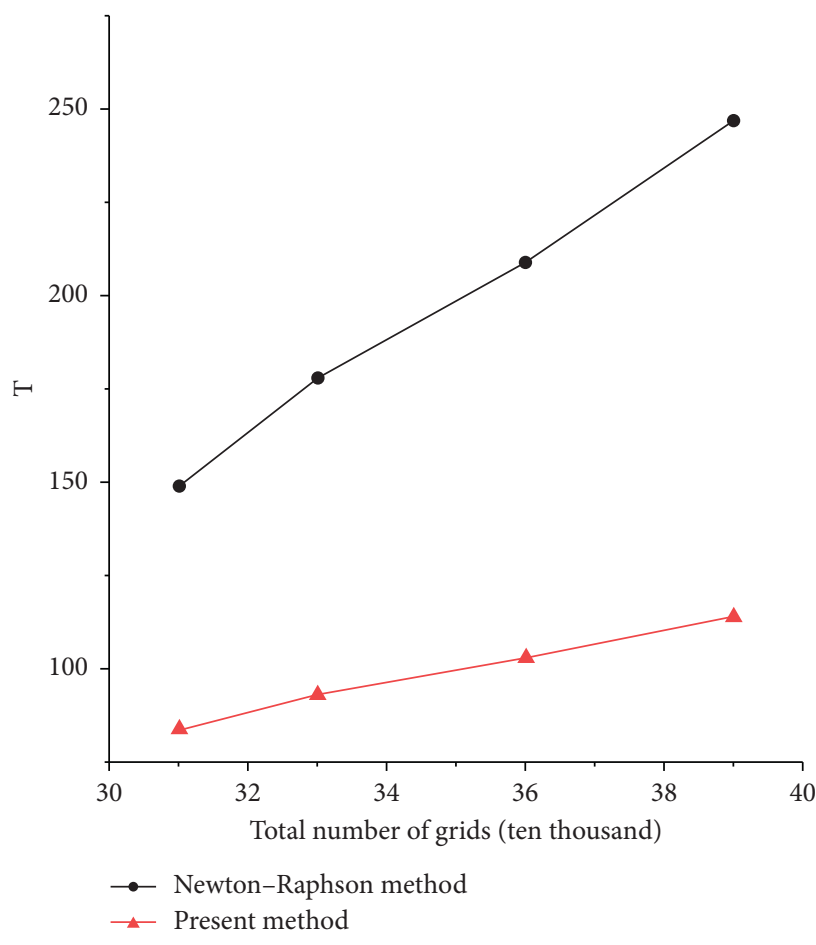

(b)

FIGURE 7: Comparison results of convergence by different methods.

that, under the condition that the accuracy of the grid is improved, the calculation accuracy and efficiency of the modified projection method in this paper are significantly improved. It also shows that the calculation result of the method in this paper is highly dependent on the grid.

\section{Conclusion}

In this paper, according to the characteristics of flexible membrane structure undergoing large deformation, the traditional projection method is modified to solve the above strong coupling integral equation, which is applied to the two-dimensional fluid-solid coupling problem and the calculation of the wind-induced fluid-structure interaction of the three-dimensional flexible membrane structure. The main conclusions are as follows:

(1) The modified projection method can be used to calculate the wind-induced fluid-structure coupling of flexible membrane structures, and its calculation accuracy and efficiency are higher than the traditional Newton-Raphson method.

(2) The modified value of the modified projection method affects the results a little, while the calculation of iteration times is an important factor affecting the results.

(3) The calculation result of the modified projection method is highly dependent on the grid, and the accuracy can be greatly improved by increasing the number of iterations in the computation.

\section{Data Availability}

The data used to support the findings of this study are included within the article.

\section{Conflicts of Interest}

The authors declare that they have no conflicts of interest.

\section{Acknowledgments}

This study was supported by the National Natural Science Foundation of China (No. 51108345), Guangxi Key Laboratory of Geomechanics and Geotechnical Engineering (GUIKENENG19-Y-21-2), Guangxi Key Laboratory of New Energy and Building Energy Saving Foundation (Gui Keneng 19-J-21-14), Open Foundation of Guangxi Key Laboratory of Embedded Technology and Intelligence (No. 2019-02-08), Joint Cultivation Program of National Natural Science Foundations of Guangxi (2019GXNSFAA245037), Guangxi Youth Innovative Talents Research Project (Guike AD19245012), and Guangxi Key Laboratory Fund of Embedded Technology and Intelligent System, Scientific and Technology Startup Foundation of Guilin University of Technology (GUTQGJJ2019042 and GUTQDJJ2019041).

\section{References}

[1] H. G. Matthies and J. Steindorf, "Partitioned strong coupling algorithms for fluid-structure interaction," Computers \& Structures, vol. 81, no. 8-11, pp. 805-812, 2003. 
[2] J. Degroote, K.-J. Bathe, and J. Vierendeels, "Performance of a new partitioned procedure versus a monolithic procedure in fluid-structure interaction," Computers \& Structures, vol. 87, no. 11-12, pp. 793-801, 2009.

[3] C. Habchi, S. Russeil, D. Bougeard et al., "Partitioned solver for strongly coupled fluid-structure interaction," Computers \& Fluids, vol. 71, pp. 306-319, 2013.

[4] A. Michalski, E. Haug, J. Bradatsch, and K.-U. Bletzinger, "Virtual design methodology for lightweight structures aerodynamic response of membrane structures," International Journal of Space Structures, vol. 24, no. 4, pp. 211-221, 2009.

[5] A. Michalski, P. D. Kermel, E. Haug, R. Löhner, R. Wüchner, and K.-U. Bletzinger, "Validation of the computational fluidstructure interaction simulation at real-scale tests of a flexible 29 m umbrella in natural wind flow," Journal of Wind Engineering and Industrial Aerodynamics, vol. 99, no. 4, pp. 400-413, 2011.

[6] M. Glück, M. Breuer, F. Durst, A. Halfmann, and E. Rank, "Computation of wind-induced vibrations of flexible shells and membranous structures," Journal of Fluids and Structures, vol. 17, no. 5, pp. 739-765, 2003.

[7] Y. Wu, Study on Wind-Induced Dynamic Response of Cable Membrane Structure with Fluid-Solid Coupling, Harbin Institute of Technology, Harbin, China, 2003.

[8] X. Sun, Study Onfluid-Structure Interaction in Wind-Induced Vibration Response of Thin-Film Structures, Harbin Institute of Technology, Harbin, China, 2007.

[9] E. Hachem, S. Feghali, R. Codina, and T. Coupez, "Anisotropic adaptive meshing and monolithic variational multiscale method for fluid-structure interaction," Computers \& Structures, vol. 122, pp. 88-100, 2013.

[10] C. Michler, S. J. Hulshoff, E. H. van Brummelen, and R. de Borst, "A monolithic approach to fluid-structure interaction," Computers \& Fluids, vol. 33, no. 5-6, pp. 839-848, 2004.

[11] S. Bailoor, A. Annangi, J. H. Seo, and R. Bhardwaj, "A fluidstructure interaction solver for compressible flows with applications in blast loading on thin elastic structures," Applied Mathematical Modelling, vol. 52, pp. 470-492, 2017.

[12] R. Bhardwaj and R. Mittal, "Benchmarking a coupled immersed-boundary-finite-element solver for large-scale flowinduced deformation," AIAA Journal, vol. 50, no. 7, pp. 1638-1642, 2012.

[13] F.-B. Tian, H. Dai, H. Luo, J. F. Doyle, and B. Rousseau, "Fluid-structure interaction involving large deformations: 3D simulations and applications to biological systems," Journal of Computational Physics, vol. 258, pp. 451-469, 2014.

[14] S. Bailoor, A. Annangi, J. H. Seo, and R. Bhardwaj, "Fluidstructure interaction solver for compressible flows with applications to blast loading on thin elastic structures," Applied Mathematical Modelling, vol. 52, pp. 470-492, 2017.

[15] M. Furquan and S. Mittal, "Flow past two square cylinders with flexible splitter plates," Computational Mechanics, vol. 55, no. 6, pp. 1155-1166, 2015.

[16] F. Sun and M. Gu, "A numerical solution to fluid-structure interaction of membrane structures under wind action," Wind \& Structures, vol. 19, no. 1, pp. 35-58, 2014.

[17] J. H. Kim and Y. H. Kim, "A predictor-corrector method for structural nonlinear analysis," Computer Methods in Applied Mechanics and Engineering, vol. 191, no. 8-10, pp. 959-974, 2001.

[18] J. L. Guermond, P. Minev, and J. Shen, "An overview of projection methods for incompressible flows," Computer
Methods in Applied Mechanics and Engineering, vol. 195, no. 44-47, pp. 6011-6045, 2006.

[19] S. Turek and J. Hron, "Proposal for numerical benchmarking of fluid-structure interaction between an elastic object and laminar incompressible flow," in Fluid-structure InteractionModeling, Simulation, Optimization, H.-J. Bungartz and M. Schafer, Eds., Springer, Berlin, Germany, 2006pp. 371-385, vol. 53 Lecture Notes in Computational Science and Engineering. 